\title{
Surface Potential Decay of Gamma-Irradiated Low-Density Polyethylene
}

\author{
George Chen \\ School of Electronics and Computer Science \\ University of Southampton, United Kingdom \\ Email: gc@ecs.soton.ac.uk
}

\begin{abstract}
In the present paper the surface potential decay after dc corona charging of gamma-irradiated low-density polyethylene (LDPE) has been studied. The irradiation was carried out in air, nitrogen gas and vacuum to investigate the effect of irradiation environment on the surface potential of LDPE. Samples with different absorbed doses were obtained by exposing samples to ${ }^{60} \mathrm{Co}$ for various times at room temperature. It has been found that there is a significant change in the surface potential decay characteristics after different irradiation dose and the surface potential decay pattern depends also on irradiation environment. The possible surface potential decay mechanisms responsible for the above were discussed. The results are useful in the context of long term performance of electrets.
\end{abstract}

\section{INTRODUCTION}

Polyethylene (PE) has been used widely as an insulation in electrical and electronic engineering. However, it has been found that the dielectric property of PE is altered in response to gamma-ray irradiation [1-3], for example, bulk charge trapping characteristics change significantly after gammairradiation and these changes are also related to irradiation environments. It can be attributed to traps or defects induced in the process of irradiation. The formation of these defects can be in the amorphous phase or on the surface of the crystallites [2]. Our previous work has pointed out that the trapping characteristic of LDPE irradiated with low dose levels $(10 \mathrm{kGy})$ is remarkably different from that irradiated with high dose levels (from $50 \mathrm{kGy}$ to $500 \mathrm{kGy}$ ), which is ascribed to the occurrence of carbonyl group $(\mathrm{C}=\mathrm{O})$ generated by radiation-induced oxidation reactions [4]. However, the influence of gamma-irradiation on surface charge behaviours is less studied. Das-Gupta [5] has reported that surface charge density on LDPE irradiated by gamma-ray with $60 \mathrm{kGy}$ is reduced by a factor of two compared with that of the unirradiated LDPE. It was suggested that if the materials is subjected to appropriate dosage of gamma-ray irradiation, their capacities of surface charge are weakened [5].

In the present paper the surface potential decay after dc corona charging of gamma-irradiated low-density polyethylene (LDPE) has been studied. The irradiation was carried out in air, nitrogen gas and vacuum to investigate the effect of irradiation environment on the surface potential of LDPE. Samples with different absorbed doses were obtained by exposing samples to ${ }^{60} \mathrm{Co}$ for various times at room temperature. The results obtained show that the behavior of surface charge is remarkably changed as a function of radiation dose and irradiation environments.

\section{EXPERIMENTAL METHOD}

\section{A. Sample preparation}

To minimise the morphological differences and charge trapping that may arise from the presence of additives, $100 \mu \mathrm{m}$ additive free LDPE film was used in the present study. LDPE samples were cut from the film, cleaned with alcohol and subsequently placed in a ${ }^{60} \mathrm{Co}$ gamma source for irradiation to different doses between $5 \mathrm{kGy}$ and $500 \mathrm{kGy}$ at a dose rate of approximately $1 \mathrm{kGy} / \mathrm{h}$. Different batches of samples were irradiated at room temperature in ambient air, oxygen-free nitrogen and vacuum. For irradiation in nitrogen and vacuum, the samples were placed inside glass tubes which were evacuated for several hours before being sealed (vacuum) or backfilled with nitrogen gas and sealed.

\section{B. Corona charging and surface potential measurement}

LDPE film was charged in a conventional corona setup as shown in Figure 1. The system consists of a high voltage needle, a wire mesh grid and an earth plate. The separation distance between the needle and grid is about $35 \mathrm{~mm}$ while the distance between the grid and sample surface is about $16 \mathrm{~mm}$. The initial surface potential of corona charged film is controlled by the grid voltage applied across the needle and the rotatable earth electrode. Corona charging time can be varied. As the main purpose of the present study is to investigate the influence of gamma irradiation and irradiation environment on surface potential decay, the charging time was fixed for two minutes. The first surface potential reading was taken about two seconds later after charging completion.

After corona charging, the sample was moved to the static monitor (Compact JCI 140) quickly for the surface potential decay observation. The monitor was about $18 \mathrm{~mm}$ above the sample. The readings from the static monitor are proportional to the value of surface potential. After a calibration these readings can be easily converted into the surface potential. The negative corona charging was used in this study and the readings from the monitor were converted to absolute potential value in all the results. All experiments were carried out under a controlled environment where temperature and relative humidity were $21^{\circ} \mathrm{C}$ and $45 \%$, because both the 
temperature and relative humidity have an influence on charge decay.

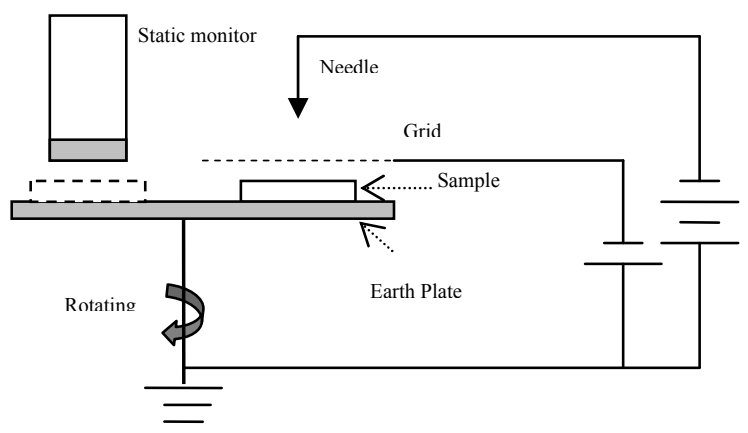

Figure 1 Corona charging and potential decay measurement system

\section{RESUltS AND DiscUSSION}

To understand the effect of gamma irradiation on surface potential, fresh LDPE samples without irradiation were corona charged under different grid voltage and their potential decay was observed for $\sim 10$ minutes. The potential decay result of corona-charged film under different charging voltages for 2 mins is shown in Figure 2. Surface potential shows an expected monotonic decay with time. However, we can observe clearly the crossover phenomenon; i.e. the surface potential in the sample with an initial high potential decays faster than that with a lower surface potential.

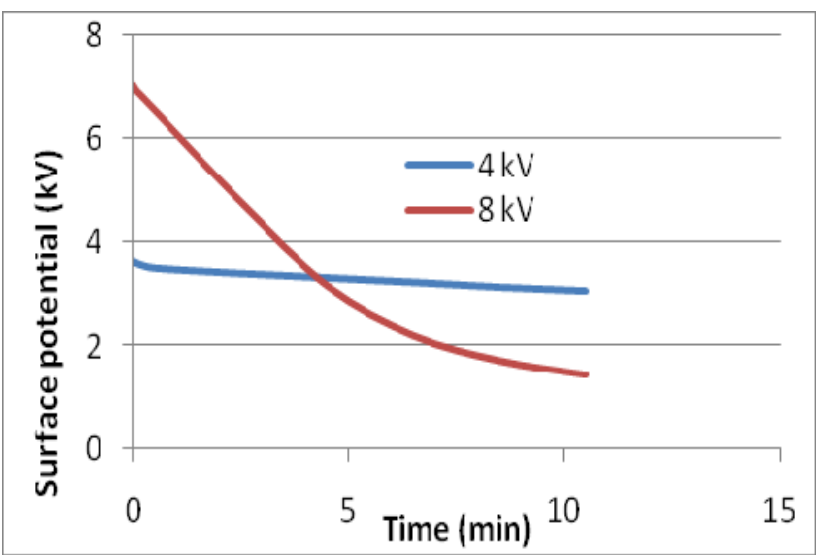

Figure 2 Surface potential decay of fresh LDPE samples after corona-charged at 4 and $8 \mathrm{kV}$.

The crossover phenomenon was first observed by Ieda et al [6] in 1967 in LDPE and subsequently observed in other materials as well. Many attempts have been made over the years to interpret the surface charge leakage and crossover phenomenon and different mechanisms have been proposed. The decay has been attributed to various mechanisms including recombination with opposite ions in air, surface migration and bulk conduction. The bulk process has been widely accepted and several models have been proposed to explain the surface potential decay [7-10]. However, the proposed explanation of the crossover phenomenon is not satisfactory. Recently, using multilayer and together with space charge measurement technique [11-12], it is clearly demonstrated that the surface potential crossover phenomenon is related to the bipolar charge injection. Simulation results from the new model based on the bipolar charge injection reveal that surface potential decay fast when the initial surface potential is higher.

As there is little change in the sample corona-charged at $4 \mathrm{kV}$, only the results from samples corona-charged at $8 \mathrm{kV}$ will be presented. Figure 3 shows the surface potential decay in samples irradiated for various doses in air. Initial observation is that by irradiation, the surface potential decay rate becomes slower, meaning the irradiated sample holding charge better. The improvement increases initially with the dose absorbed and reaches its peak around $25 \mathrm{kGy}$. Further increase in irradiation dose leads to a fast potential decay. The surface potential at the end of decay measurement as a function of irradiation dose is shown in Figure 4. An attempt has been made to corona charging the sample exposed to $500 \mathrm{kGy}$, however, the sample was very brittle and became difficult to handle without breaking it.

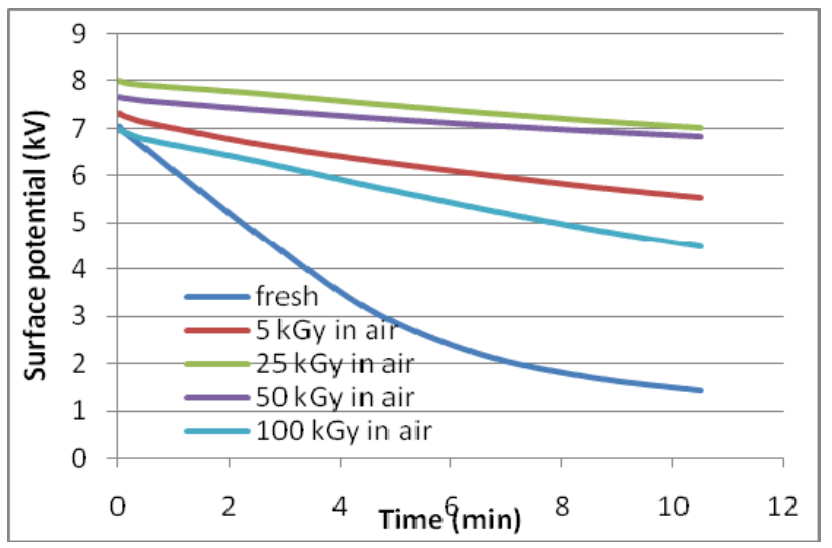

Figure 3 Surface potential decay in irradiated LDPE samples (in air) after corona-charged at $8 \mathrm{kV}$.

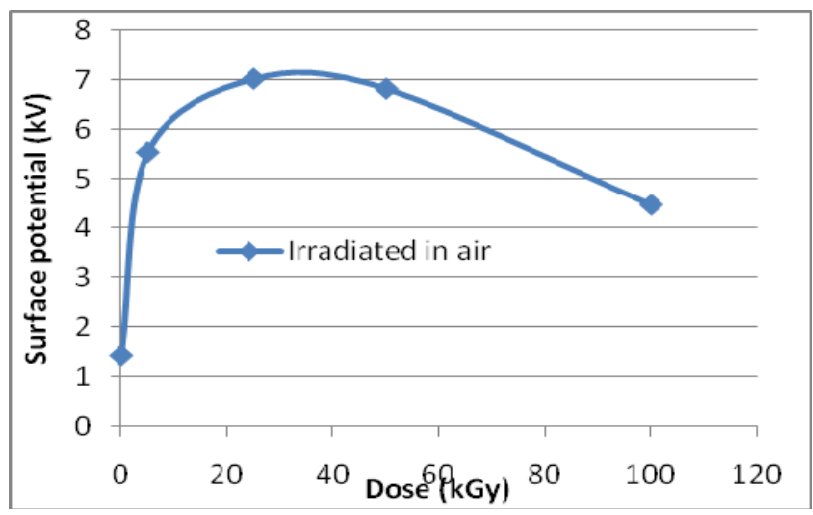

Figure 4 Surface potential after 10 min decay as a function of irradiation dose.

It is evident that gamma irradiation can slow down surface potential decay. However, our results seem to contradict with 
those in [5] where the initial surface potential of polypropylene (PP) irradiated to $60 \mathrm{kGy}$ in nitrogen gave a much lower value compared with the unirradiated sample. However, the surface charge decay in the case of PP seems unaffected by irradiation. From the view point of electrets and their applications, this is good news as charge stability has been improved significantly by gamma irradiation. This will lead to a long lifetime for the devices.

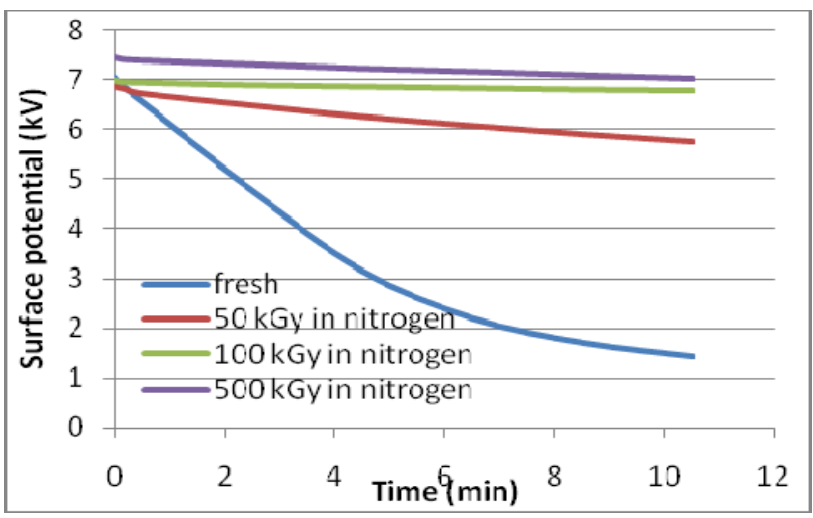

Figure 5 Surface potential decay in irradiated LDPE samples (in nitrogen) after corona-charged at $8 \mathrm{kV}$.

Figure 5 illustrates surface potential decay when samples were irradiated in oxygen free nitrogen gas. Again, after gamma irradiation the surface charge decay becomes slower. Differing from the samples irradiated in air, the improvement continues even upto $500 \mathrm{kGy}$.

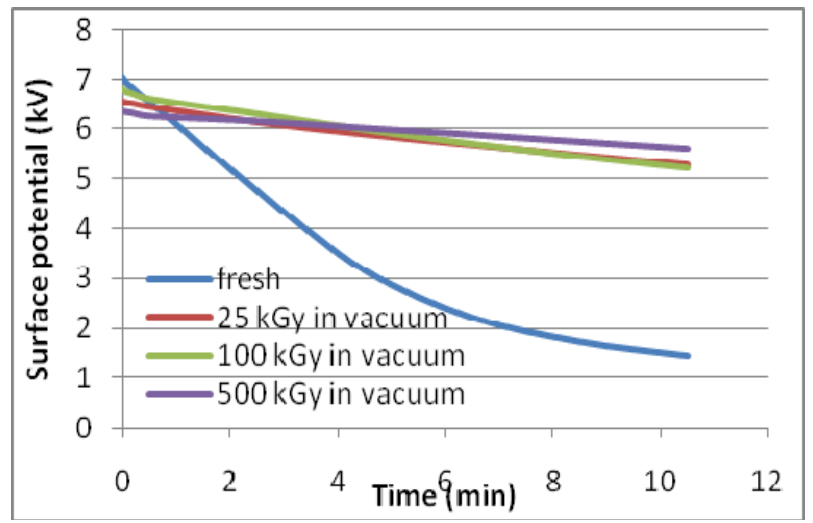

Figure 6 Surface potential decay in irradiated LDPE samples (in vacuum) after corona-charged at $8 \mathrm{kV}$.

Figure 6 shows the surface potential decay of samples irradiated in vacuum for various doses. Similar to those irradiated in nitrogen gas, the surface potential decays much slower after irradiation in vacuum. However, the dose effect on the surface potential decay is not obvious above $25 \mathrm{kGy}$. Figure 7 summarises the results from samples irradiated to 100 kGy in various environments together with the surface potential decay from fresh sample. It is evident that irradiation in nitrogen gas has the most effect on the surface potential decay, followed by irradiation in vacuum.

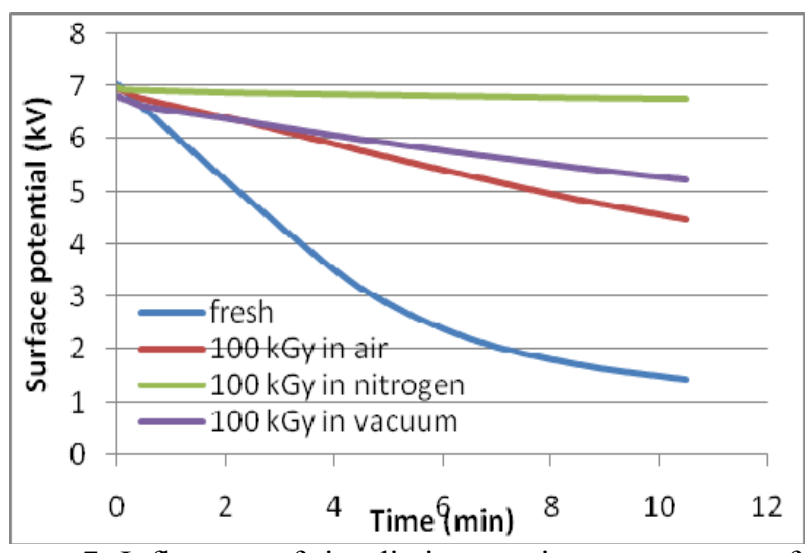

Figure 7 Influence of irradiation environment on surface potential decay.

When a material is subject to radiation high energy can cause chemical changes such as crosslinking and chain scission. The exact chemical reaction depends on polymeric material and its environment. The detailed process may described as the following: Ionization of the material gives rise to radicals and to the subsequent alteration of the structure of the material via chemical radical reactions and the mechanisms of cross-linking, chain scission, gas production and oxidation cited earlier. Thus as well as releasing charge within the material, radiation causes it to change from its original state. This happens not only as irradiation takes place, but also afterwards as oxygen, for example, diffuses into the bulk and produces oxidation products such as ketones, alcohols and hydroperoxides among many others, depending on the material in question.

It is generally agreed that the main reaction in polyethylene is crosslinking especially at lower doses. As polyethylene is a semicrystalline material. In addition to chemical reaction, it is also possible that some extra defects will be generated in the crystalline region when subjected to high energy irradiation. The defect generation will occur irrespective of irradiation environment. In the presence of oxygen, one oxidation product that is readily observable with irradiated LDPE is the carbonyl group $(\mathrm{C}=\mathrm{O})$. It is well established that the concentration of carbonyl groups increases with radiation dose and that the carbonyl groups generate a shallow trap that readily assists charge transport [13].

In the absence of oxygen such as in nitrogen gas or vacuum, LDPE undergoes predominantly cross-linking under irradiation. From its effect on the surface potential decay, it seems that there are some differences between irradiation in nitrogen and vacuum. It is not know at this stage that the differences are chemical or physical in nature.

Surface potential decay following corona charging involves two processes, i.e. charge transfer from surface to the bulk and charge transport through the bulk. Our previous research [14] on space charge behavior in irradiated LDPE samples reveals that irradiation generally leads to a slow decay, indicating the formation of deep traps after irradiation. Most importantly, it has 
been observed that space charge patterns for the samples irradiated to $100 \mathrm{kGy}$ are very different depending on irradiation environment. Dominated positive charge is present in the bulk in the case of irradiated in air. On the contrary, only negative charge is observed in the case of sample irradiated in nitrogen gas. Both positive and negative charges are found in the case of sample irradiated in vacuum. Figure 8 shows the bulk charge decay after the removal of the applied voltage following the application of an electric field of $50 \mathrm{kV} / \mathrm{mm}$ for 60 minutes.

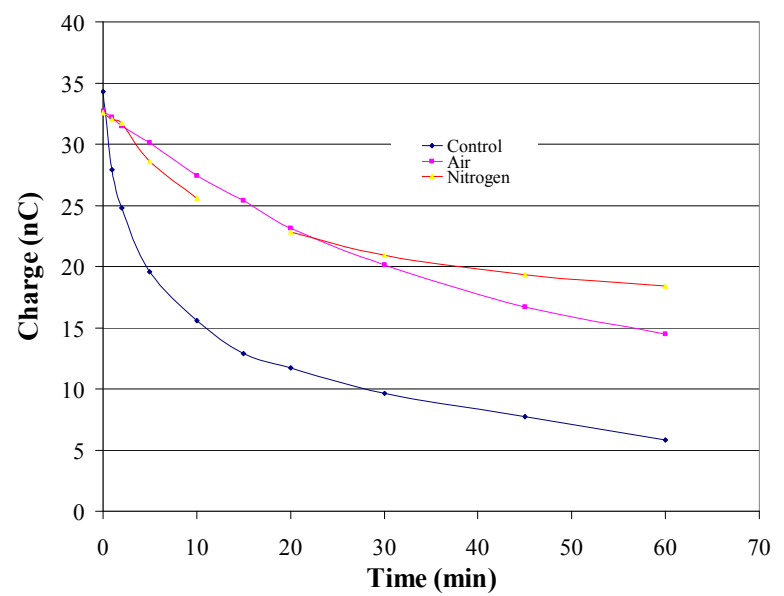

Figure 8 Charge decay rate for samples irradiated to $100 \mathrm{kGy}$ under different environments.

From Figure 8 it can be seen that the sample irradiated in nitrogen gas shows an overall slow decay rate. This is in agreement with the observation from surface potential decay.

The surface of the sample may also be modified by gamma irradiation. If deep traps are formed due to irradiation then the charge transfer from the surface to the bulk can be slowed down, leading to a slow potential decay. Das-Gupta measured surface current of gamma irradiated LDPE and found that the surface current decreases with the dose absorbed by the material [2]. This may indicate the surface states created by irradiation form deep trap for charges, leading to a low surface current. In the present study it is difficult to separate individual contribution of bulk and surface towards the slow potential decay. Further research will be carried out to identify the key factor that affects potential decay as it has an important impact on the improvement of charge stability. In addition, it would be interesting to see the influence of gamma irradiation on surface potential decay when positive corona is adopted.

\section{CONCLUSIONS}

In the present paper the influence of gamma irradiation on the surface potential of LDPE has been examined. The following conclusions may be drawn:

The surface potential decay is significantly affected by gamma irradiation. For the sample irradiated in air, the decay rate is a function of the absorbed dose and the slowest decay rate is around $25 \mathrm{kGy}$. There are marked differences in the surface potential decay rate between the samples irradiated in air in comparison with LDPE irradiated in either vacuum or oxygenfree nitrogen gas. It is evident that the presence of oxygen during irradiation makes the surface potential decay fast compared with those without oxygen. The sample irradiated in nitrogen gas gives the slowest decay rate.

It is difficult to pin point the mechanism but deep traps introduced by irradiation are the potential candidate for the slow decay. There is some experimental evidence of formation of deep traps in the bulk following gamma irradiation but the effect of surface contribution can't be ruled out.

The results obtained are significant in the context of charge stability of electrets. It seems that the long term characteristics of electrets can be improved by irradiation.

\section{REFERENCES}

[1] G. Chen, A. E. Davies and H. M. Banford, "Influence of radiation environments on space charge formation in $\gamma$-irradiated LDPE", IEEE Trans. Dielectr. Electr. Insul., Vol. 6, No. 6, pp. 882-886, 1999.

[2] D. K. Das-Gupta, "Electrical properties of surfaces of polymeric insulators", IEEE Trans. Electr. Insul., Vol. 27, No. 5, pp. 909-923, 1992.

[3] G. Chen, H. M. Banford and A. E. Davies, "Space charge formation in $\gamma$-irradiation low density polyethylene", IEEE Trans. Dielectr. Electr. Insul., Vol. 5, No. 1, pp. 51-57, 1998.

[4] M. Fu, G. Chen, L. A. Dissado, J. C. Fothergill and C. Zou, "The effect of gamma irradiation on space charge behaviour and dielectric spectroscopy of low-density polyethylene". In: 2007 IEEE International Conference on Solid Dilectrics, 8th -- 13th July 2007, Winchester, UK. pp. 442-445, 2007.

[5] D. K. Das-Gupta and W. F. Schmidt, "Electrical properties of surfaces of unirradiated and irradiated polymers in humid environments", IEEE Trans. Components, Packaging and Manufacturing Technology, Vol. 18, No. 2, pp. 266-269, 1995.

[6] M. Ieda, G. Sawa and U. Shinohara, "A decay process of surface electric charges across polyethylene film”, Jpn. J. Appl. Phys. Vol. 6, pp. $793-794,1967$.

[7] T. J. Sonnonstine and M. M. Perlman, Surface-Potential Decay in Insulators with Fielddependent Mobility and Injection Efficiency, J. App. Phys. vol. 46, p. 3975 - 3981, 1975.

[8] R. Toomer and T. J. Lewis, Charge Trapping in Corona-Charged Polyethylene Films, J. Phys. D: Appl. Phys. vol. 13, p. 1343-1356, 1980.

[9] H. von. Berlepsch, Interpretation of Surface Potential Kinetics in HDPE by a Trapping Model, J.Phys. D: Appl. Phys. Vol. 18, p. 1155 - 1170, 1985.

[10] H. J. Wintle, "Surafce-charge decay in insulators with non-constant mobility and with deep trapping", J. Appl. Phys. Vol. 43, pp. $2927-$ 2930, 1972.

[11] Y. Takai, K. Mori, T. Mizutani and M. Ieda, "Investigation of traps in gamma-irradiated polyethylene by photostimulated detrapping current analysis" Jpn. J. Appl. Phys. Vol. 15, pp 2341-2347, 1976.

[12] G. Chen, Z. Xu and L. W. Zhang, "Measurement of the surface potential decay of corona-charged polymer films using the pulsed electroacoustic method", Meas. Sci. Technol. Vol. 18, pp. 1453-1458, 2007.

[13] Z. Xu, L. Zhang and G. Chen, "Decay of electric charge on corona charged polyethylene", J. Phys. D: Appl. Phys. Vol. 40, pp. 7085$7089,2007$.

[14] G. Chen and M. Fu, "The Influence of Gamma Irradiation on Space Charge in LDPE". In: 8th International Conference on Properties and Applications of Dielectric Materials, 26 - 30 June 2006, Bali, Indonesia. pp. 131-134, 2006. 